\title{
Effects of psychostimulant
}

withdrawal on latent inhibition of

conditioned active avoidance and

prepulse inhibition of the acoustic startle response

\author{
Journal Article \\ Author(s): \\ Murphy, Carol A.; Di lorio, Lucia; Feldon, Joram \\ Publication date: \\ 2001 \\ Permanent link: \\ https://doi.org/10.3929/ethz-b-000422995
}

Rights / license:

In Copyright - Non-Commercial Use Permitted

Originally published in:

Psychopharmacology 156(2-3), https://doi.org/10.1007/s002130100759 
Carol A. Murphy $\cdot$ Lucia Di Iorio · Joram Feldon

\section{Effects of psychostimulant withdrawal on latent inhibition of conditioned active avoidance and prepulse inhibition of the acoustic startle response}

Received: 19 September 2000 / Accepted: 24 February 2001 / Published online: 14 June 2001

(C) Springer-Verlag 2001

\begin{abstract}
Rationale: Chronic intermittent administration of amphetamine and cocaine can precipitate psychotic episodes in humans and produce persistent behavioral changes (i.e. increased locomotion, stereotypy) in the rat. The psychostimulant sensitization model of psychosis holds that the repeated administration of drugs such as amphetamine and cocaine induces long-lasting neuroadaptations and behavioral outcomes in animals that parallel aspects of the schizophrenic condition. Objectives: In the present study, we attempted to validate this model further by examining the effects of short-term withdrawal from repeated administration of cocaine and amphetamine on performance in two animal behavioral models of cognitive deficits found in schizophrenia: latent inhibition and prepulse inhibition. Reductions in both of these behavioral phenomena have been reported in schizophrenic patients and in acutely amphetamine-treated rats. Methods: Animals were tested after 4 days of withdrawal from 5 days of daily systemic $20 \mathrm{mg} / \mathrm{kg}$ cocaine or $1.5 \mathrm{mg} / \mathrm{kg}$ amphetamine injections for either latent inhibition of two-way active avoidance acquisition or prepulse inhibition of an acoustic startle response. Results: Our results indicate that, rather than reducing the expression of these behaviors, withdrawal from either cocaine or amphetamine enhanced the expression of latent inhibition of the active avoidance response while having no effect on prepulse inhibition of acoustic startle. Conclusions: These data indicate that although the sensitized response to amphetamine and cocaine administration may model some aspects of schizophrenic psychosis, behaviors exhibited by sensitized animals in the absence of an acute drug challenge are not consistent with models of the positive symptoms of schizophrenia.
\end{abstract}

C.A. Murphy $(\bowtie) \cdot$ L. Di Iorio $\cdot$ J. Feldon Laboratory of Behavioral Neurobiology,

Swiss Federal Institute of Technology (ETH-Zurich),

Schorenstrasse 16, 8603 Schwerzenbach, Switzerland e-mail: murphy@ toxi.biol.ethz.ch

Tel.: +41-1-6557368, Fax: +41-1-6557203
Keywords Amphetamine - Cocaine - Latent inhibition . Prepulse inhibition · Withdrawal $\cdot$ Schizophrenia

\section{Introduction}

Both amphetamine and cocaine can induce behavioral states which resemble the psychotic episodes observed in acutely schizophrenic patients (Snyder 1973; Post 1975; Brady et al. 1991). This observation has led to the development of the psychostimulant sensitization model of schizophrenia, in which the enhanced locomotor and stereotypic responses of rodents to repeated psychostimulant administration are studied for their relevance to the positive symptoms of schizophrenia (i.e. delusions, hallucinations, paranoia) in humans (Robinson and Becker 1986; Pierce and Kalivas 1997). In contrast to the effects of repeated drug administration, the abrupt termination of chronic psychostimulant intake by human drug users can result in symptoms of dysphoria, anhedonia, anxiety and drug craving (Gawin and Kleber 1986; Gawin 1991; Lago and Kosten 1994). Such symptoms can also be effectively modelled in the psychostimulant-withdrawn rat (Leith and Barrett 1976; Markou and Koob 1991; Harris and Aston-Jones 1993; Sarnyai et al. 1995; Borowski and Kokkinidis 1998; Barr et al. 1999; Barr and Phillips 1999; Murphy et al. 2001) and have been postulated to contribute to the high frequency of relapse among recently-withdrawn addicts.

The presence of altered behavioral states during withdrawal from psychostimulant administration reflects functional changes in the response of the nervous system to stimuli other than the previously administered drug. Although such changes may be more in line with the symptoms of anhedonia and increased anxiety that characterize psychostimulant withdrawal than with the positive symptoms of schizophrenia which repeated drug administration is believed to simulate, it would be useful to examine further the cognitive consequences of psychostimulant withdrawal in the rat as these may be relevant to the schizophrenic condition. Two animal behavioral 
models believed to reflect cognitive/attentional deficits typical of schizophrenic patients are latent inhibition (LI) of classically-conditioned responding and prepulse inhibition (PPI) of the startle response.

LI is the process by which repeated non-reinforced pre-exposure to a conditioned stimulus (CS, e.g. tone) retards conditioning to the same stimulus when it is subsequently paired with reinforcement (unconditioned stimulus (UCS), e.g. shock; Lubow 1973). PPI is the normal reduction in response amplitude to a startling stimulus when it is immediately preceded by a less intense, non-startling stimulus and is believed to represent an operational measure of sensorimotor gating ability (Graham 1975; Hoffman and Ison 1980; Koch and Schnitzler 1997). Reductions in both LI and PPI have been reported in schizophrenic patients (Braff et al. 1978; Baruch et al. 1988; Braff and Geyer 1990; Geyer et al. 1990; Gray et al. 1992; 1995; but see Swerdlow et al. 1996). These deficits in PPI and LI may reflect, respectively, the sensorimotor gating impairments and the inability to ignore irrelevant stimuli which are characteristic of many schizophrenic patients. In rats, the administration of low doses of amphetamine during CS pre-exposure and CS-UCS conditioning attenuates LI, whereas both typical and atypical antipsychotic drugs antagonize amphetamine-induced disruptions in LI and potentiate LI when administered alone (Weiner et al. 1984, 1996; Weiner and Feldon 1987; Dunn et al. 1993). PPI is similarly reduced in rats following activation of the DA mesolimbic system and these reductions are likewise reversed by antipsychotic drug pretreatment (Mansbach et al. 1988; Swerdlow et al. 1992; Taylor et al. 1995). Thus, there is considerable clinical and experimental evidence linking dopaminergic (DA) hyperactivity to disruptions of LI and PPI.

Long-term exposure to psychostimulants reportedly invokes neurochemical adaptations in the brain that oppose the pharmacological effects of the drug and may persist after termination of drug intake, causing the state of withdrawal (Koob and Bloom 1988). Consistent with this idea are studies linking the psychostimulant withdrawal syndrome to low brain DA activity in both humans and rats (Parsons et al. 1991; Imperato et al. 1992; Rossetti et al. 1992; Weiss et al. 1992, 1997; Lago and Kosten 1994; Kuhar and Pilotte 1996). The presence of decreased accumbens DA during psychostimulant withdrawal would likely produce a contrasting pattern of changes in LI and PPI to the DA-agonistic effects of acute psychostimulant administration.

Although recent reports have indicated a lack of influence of prior psychostimulant administration on PPI (Druhan et al. 1998; Martinez et al. 1999), there are suggestions that PPI can be influenced by a history of psychoactive drug intake under specific experimental conditions (Zhang et al. 1998; Martin-Iverson 1999; Byrnes and Hammer 2000). The effects of withdrawal from chronic psychostimulant treatment on LI have not been well investigated; however, we recently found an enhancement in the expression of LI of conditioned freez- ing behavior in animals withdrawn from 5 days of cocaine administration (Murphy et al. 2001). In the present study we tested the generalizability of this finding to a second behavioral paradigm, LI of a two-way active avoidance response. Animals administered cocaine or amphetamine once daily for 5 days were tested after 4 days of withdrawal from drug treatment for the expression of LI of two-way active avoidance and PPI of the acoustic startle response. Animals were subsequently tested with a challenge injection of drug to assess their degree of locomotor sensitization.

\section{Materials and methods}

\section{Subjects}

Male Wistar rats [Zur: WIST (HanIbm); 250-350 g] obtained from our in-house specific-pathogen-free (SPF) breeding facility were used as subjects in these experiments. Animals were singly housed in a temperature $\left(21 \pm 1^{\circ} \mathrm{C}\right)$ and humidity $(55 \pm 5 \%)$ controlled animal facility and maintained on a reversed 12:12 lightdark cycle (lights off at 7:00 a.m.) with ad libitum access to food and water. All procedures were carried out during the dark phase of the light-dark cycle. These procedures were performed in accordance with the regulations of the Swiss Federal Veterinary Office.

Drugs

$d$-Amphetamine sulfate (Research Biochemicals International, Natick, Mass., USA) and cocaine hydrochloride (Firma Anseler AG, Herisau, Switzerland) were dissolved in a $0.9 \% \mathrm{NaCl}$ solution to obtain concentrations of $1.5 \mathrm{mg} / \mathrm{ml}$ amphetamine (calculated as the salt) and $20 \mathrm{mg} / \mathrm{ml}$ cocaine, respectively. Vehicle-treated groups received $0.9 \% \mathrm{NaCl}$ solution. All solutions were administered IP in a volume of $1 \mathrm{ml} / \mathrm{kg}$. The cocaine and amphetamine dosing schedules used in this study were chosen on the basis of previous work in our laboratory demonstrating robust locomotor sensitization to subsequent drug challenge following these pretreatments.

\section{Apparatus}

\section{Two-way active avoidance}

Four identical two-way shuttle boxes (Coulbourn Instruments, model E10-16TC), each set in a ventilated, sound-insulated isolation cubicle (Coulbourn Instruments, model E10-20) were used in tests of two-way active avoidance behavior. Each box was $35 \mathrm{~cm}$ long, $17 \mathrm{~cm}$ deep, and measured $21.5 \mathrm{~cm}$ in height from the raised grid floor. A 4-cm high aluminum hurdle served as a barrier that separated the box into two identical compartments. The hurdle was low enough to allow the subject to shuttle freely between the two compartments, and was thin enough to ensure that the subjects could not stand on it to avoid foot shocks. The grid floor (Model E10-16RF) consisted of 24 stainless steel rods with a diameter of $0.48 \mathrm{~cm}$, spaced $1.5 \mathrm{~cm}$ apart center to center, through which scrambled foot shocks were delivered by a constant current shock generator (Coulbourn Instruments, Model E13-14) and scanner (Model E13-13) set at $0.5 \mathrm{~mA}$. The conditioned stimulus (CS) was an $85-\mathrm{dB}[\mathrm{A}]$ tone produced by a $2.9 \mathrm{kHz}$ tone module (Coulbourn Instruments, Model E12-02) placed behind the shuttle box on the floor of the isolation cubicle. Background noise was provided by a ventilation fan affixed to each isolation cubicle. 


\section{Prepulse inhibition of acoustic startle}

Prepulse inhibition (PPI) testing was conducted in four startle chambers (SR-LAB, San Diego Instruments, San Diego, Calif., USA), each consisted of a transparent Plexiglas tube (diameter $8.2 \mathrm{~cm}$, length $20 \mathrm{~cm}$ ) mounted on a Plexiglas frame within a ventilated enclosure. A speaker mounted $24 \mathrm{~cm}$ above the tube provided the acoustic noise bursts. Startle pulses consisted of $120 \mathrm{~dB}[\mathrm{~A}]$ broad band bursts of $30 \mathrm{~ms}$ duration. Prepulses, $20 \mathrm{~ms}$ of broad band burst, had intensities of either 72, 76, 80 or $84 \mathrm{~dB}[\mathrm{~A}]$. The time interval between the prepulse offset and the pulse onset was always $80 \mathrm{~ms}$. Background noise of $70 \mathrm{~dB}[\mathrm{~A}]$ was maintained throughout the session. A piezoelectric accelerometer mounted below the frame detected and transduced motion within the tube. Startle amplitudes were defined as the average of 100, 1-ms stabilimeter readings collected from stimulus onset.

\section{Locomotor activity}

Locomotor activity was assessed in circular polycarbonate test chambers $(30 \mathrm{~cm}$ diameter, $35 \mathrm{~cm}$ high walls) with sawdust bedding covering the floor. An infrared sensor unit (Coulbourn Instruments, Model E24-61) was positioned at a $15^{\circ}$ angle, $10 \mathrm{~cm}$ above the top of the chamber. Movement units detected by the sensor were transmitted through an infrared motion interface (Coulbourn Instruments, Model E91-12-421) to an infrared motion activity monitor controller/analyser (Habitest Universal Link, Coulbourn Instruments).

Experiment 1: effects of psychostimulant withdrawal on two-way active avoidance acquisition and latent inhibition

On each of 3 days prior to conducting any experimental procedures, each rat was handled for an average of $3 \mathrm{~min}$. The effects of cocaine (COC) and amphetamine (AMPH) withdrawal on latent inhibition of active avoidance acquisition were studied in comparison to independent groups of saline vehicle (VEH)-treated animals. All animals were injected IP once every day for 5 consecutive days with $20 \mathrm{mg} / \mathrm{kg}$ COC, $1.5 \mathrm{mg} / \mathrm{kg}$ AMPH or VEH. Injections were administered between 0900 and 1000 hours.

Conditioned active avoidance testing was carried out over 3 days, consisting of 2 days of pre-exposure to the test apparatus and/or tone CS and 1 day of two-way avoidance acquisition testing. The first pre-exposure session was conducted 2 days after the last vehicle or psychostimulant injection; consequently, active avoidance testing was performed after a 4-day withdrawal period. On each of the 2 pre-exposure days (sessions beginning at 1000 hours), animals were placed in the boxes for $50 \mathrm{~min}$, with the house light on continuously. Half of the animals were tone-pre-exposed (PE); that is, they experienced 50 presentations of a 10 -s-tone conditioned stimulus (CS) during each of the two pre-exposure sessions (variable inter-trial interval (ITI); mean ITI $=50 \mathrm{~s}$, range $=10-90 \mathrm{~s}$ ). The remaining half of the animals comprised the non-pre-exposed (NPE) groups, which did not hear the tone CS during either preexposure session. The number of spontaneous crosses between the two sides of the shuttle box was recorded during both pre-exposure sessions to provide a measure of basal activity level. On the avoidance test day each subject was placed in the appropriate shuttle box and 100 avoidance trials were administered, again with a variable ITI (mean $=50 \mathrm{~s}$, range $=10-90 \mathrm{~s}$ ). Each avoidance trial began with the onset of the 10-s tone CS. If a subject failed to shuttle to the other side within $10 \mathrm{~s}$ after CS onset (i.e., an avoidance response), a foot shock of $0.5 \mathrm{~mA}$ was delivered, the CS remaining on with the shock. The maximal duration of the shock was $2 \mathrm{~s}$. A shuttle during this period (i.e., an escape) terminated the shock as well as the CS. If a subject failed to shuttle during the 12 -s period, it was recorded as a non-escaped trial.

One day following active avoidance testing, all of the AMPHwithdrawn animals and half of the COC-withdrawn animals were assessed for their locomotor response to a challenge injection of either AMPH or COC, respectively. Each rat was first placed in the activity chamber for a 30-min habituation session. Subsequently, animals were injected with $0.9 \%$ saline vehicle, and placed into the chamber for an additional 30-min session. Finally, animals received a psychostimulant injection, and their locomotor response to drug was monitored for $60 \mathrm{~min}$. Animals previously tested for the effects of amphetamine withdrawal on active avoidance were administered an injection of AMPH $(1.5 \mathrm{mg} / \mathrm{kg}$, IP) and animals previously tested for the effects of cocaine withdrawal received an injection of COC $(20 \mathrm{mg} / \mathrm{kg}$, IP).

Experiment 2: effects of psychostimulant withdrawal on prepulse inhibition of acoustic startle

Solutions of AMPH, COC and VEH were administered according to the same injection schedule and in the same dosages as described for experiment 1 . Animals were tested for prepulse inhibition of acoustic startle 4 days after their last injection, the same withdrawal interval at which latent inhibition of active avoidance was measured in experiment 1 . The startle session began with a 5-min acclimatization period during which only $70 \mathrm{~dB}[\mathrm{~A}]$ background noise was presented. This background noise continued throughout the remainder of the test session. After the acclimatization period, four startle pulses of $120 \mathrm{~dB}$ [A], each of 30-ms duration, were presented to permit an initial test of startle responding prior to the onset of prepulse testing. This "warm-up" period was followed by six blocks of 11 test trials each. Each block of trials consisted of four different trial types presented pseudo-randomly throughout the whole session, i.e. pulse alone (two trials), prepulse alone (one trial for each of the four prepulse intensities), prepulse followed by a pulse (one trial for each of the four prepulse intensities) or no stimulus (one trial).

\section{Statistical analysis}

All statistical analyses were performed using the StatView statistical software package. ANOVAs with between-subjects factors of Drug treatment (COC versus VEH or AMPH versus VEH) and Pre-exposure condition (PE versus NPE) and a repeated measurements factor of Blocks of ten trials were used to analyze group differences in percentage avoidance responses. One-way factorial ANOVAs were used to analyse the effects of drug treatment on numbers of spontaneous shuttles during the pre-exposure sessions and on numbers of inter-trial interval shuttles and non-escaped trials during avoidance testing. In the prepulse inhibition test, startle amplitude to the pulse-alone trials was analyzed using ANOVAs with a between-subjects factor of Drug (COC versus VEH or AMPH versus VEH) and a within-subjects factor of either the first four or the last 12 pulse-alone Trials. The percentage inhibition of startle induced by each prepulse intensity [\% prepulse inhibition (PPI)] was calculated as: 100-(100xstartle amplitude on prepulse trial)/(mean startle amplitude on pulse alone trials). The mean percentage PPI was analyzed using a $2 \times 4$ ANOVA with a between-subjects factor of Drug (COC versus VEH or AMPH versus VEH) and a within-subjects factor of Prepulse intensity (72, 76, 80 and $84 \mathrm{~dB}[\mathrm{~A}])$. Locomotor activity data were analyzed separately for each testing session using two-way ANOVAs with a between-subjects factor of Drug (COC versus VEH or AMPH versus VEH) and a within-subjects factor of 10-min Blocks. 


\section{Results}

Experiment 1: effects of psychostimulant withdrawal on two-way active avoidance acquisition and latent inhibition

\section{Amphetamine withdrawal}

A comparison of the number of shuttles made during the two pre-exposure sessions (Table 1) revealed a significant effect of Session $[F(1,28)=27.1, P<0.0001]$, reflecting greater activity during the first pre-exposure session compared to the second, as well as a significant effect of Pre-exposure condition $[F(1,28)=5.1, P<0.05]$ such that pre-exposed (PE) animals shuttled less frequently during these sessions than non-pre-exposed animals (NPE). Amphetamine-treated (AMPH) and vehicle-treated (VEH) groups made similar numbers of shuttles during both sessions, regardless of Pre-exposure condition.

Table 1 Numbers of shuttles made during 2 days of avoidance apparatus and/or tone CS pre-exposure. Tone-pre-exposed $(P E)$ and non-pre-exposed (NPE) animals were placed in the shuttle boxes on day $2(P E$ day 1$)$ and day 3 (PE day 2$)$ of withdrawal from 5

\begin{tabular}{|c|c|c|c|c|}
\hline Avoidance experiment & Drug condition & Pre-exposure condition & PE day 1 & PE day 2 \\
\hline Amphetamine & $\begin{array}{l}\text { VEH } \\
\text { VEH } \\
\text { AMPH } \\
\text { AMPH }\end{array}$ & $\begin{array}{l}\text { NPE } \\
\text { PE } \\
\text { NPE } \\
\text { PE }\end{array}$ & $\begin{array}{l}41 \pm 7 \\
33 \pm 9 \\
46 \pm 6 \\
32 \pm 5\end{array}$ & $\begin{array}{l}32 \pm 7 \\
23 \pm 5 \\
34 \pm 3 \\
16 \pm 3\end{array}$ \\
\hline Cocaine & $\begin{array}{l}\text { VEH } \\
\text { VEH } \\
\text { COC } \\
\text { COC }\end{array}$ & $\begin{array}{l}\text { NPE } \\
\text { PE } \\
\text { NPE } \\
\text { PE }\end{array}$ & $\begin{array}{l}41 \pm 7 \\
28 \pm 4 \\
40 \pm 5 \\
23 \pm 2\end{array}$ & $\begin{array}{l}29 \pm 5 \\
22 \pm 3 \\
30 \pm 4 \\
16 \pm 2\end{array}$ \\
\hline
\end{tabular}

An analysis of percentage avoided trials during test (Fig. 1A, C) using a $2 \times 2 \times 10$ ANOVA (Drug Treatment $\times$ Pre-exposure condition $\times$ Blocks of ten trials) revealed highly significant main effects of Pre-exposure condition $[F(1,28)=12.1, P<0.002]$ and Blocks $[F(9,252)=$ 28.1, $P<0.001]$, a significant interaction of Pre-exposure condition $\times$ Blocks $[F(9,252)=2.0, P<0.05]$ and a significant interaction of Pre-exposure condition $\times$ Drug treatment $\times$ Blocks $[F(9,252)=2.1, P<0.05]$. These effects reflect the fact that latent inhibition, i.e. reduced avoidance performance in PE compared to NPE rats, was evident in AMPH- but not VEH-treated animals, a group difference which was most apparent toward the end of the avoidance test session, when the proportion of avoided trials was highest in all groups. This result was further supported by two separate $2 \times 10$ ANOVAs comparing PE and NPE groups within each drug treatment condition. An analysis confined to the AMPH groups yielded a highly significant main effect of Pre-exposure condition

days of amphetamine $(A M P H)$, cocaine $(C O C)$ or saline vehicle $(V E H)$ injections. Amphetamine experiment: $n=8$ per group. Cocaine experiment: $n=12$ per group. Values are means \pm SEM
Fig. 1 Percentage avoided trials during a 100-trial test of two-way active avoidance in animals that had been previously treated with five daily injections of $\mathbf{A}, \mathbf{B}$ saline vehicle, $\mathbf{C}$ amphetamine or $\mathbf{D}$ cocaine. All animals were tested 4 days after their last drug or vehicle injection. On each of 2 days prior to avoidance testing, animals were placed in the shuttle box for a 45-min habituation session during which time animals in the pre-exposed $(P E)$ groups were presented with 30 , 10 -s tones and animals in the non-pre-exposed (NPE) groups experienced no other stimuli. Amphetamine experiment: $n=8$ per group. Cocaine experiment: $n=12$ per group. Values are means \pm SEM
AMPHETAMINE WITHDRAWAL

A

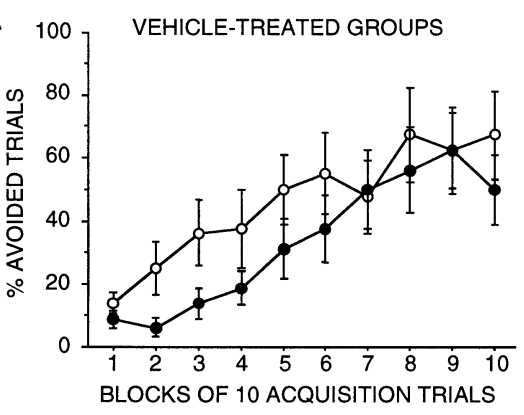

C

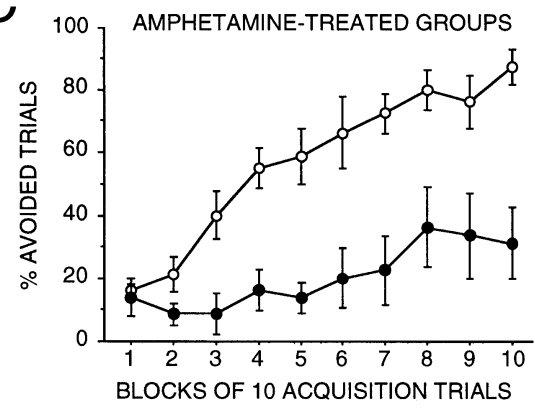

COCAINE WITHDRAWAL

B

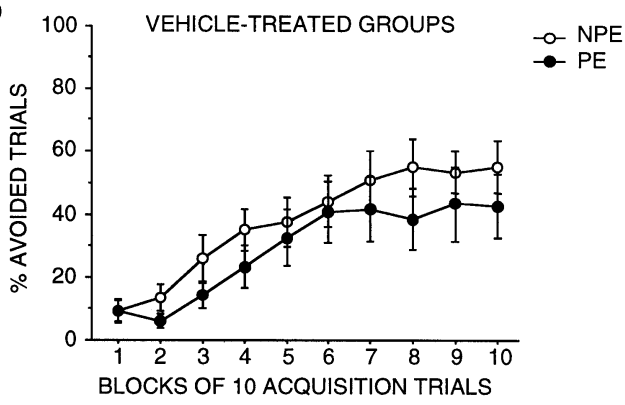

D

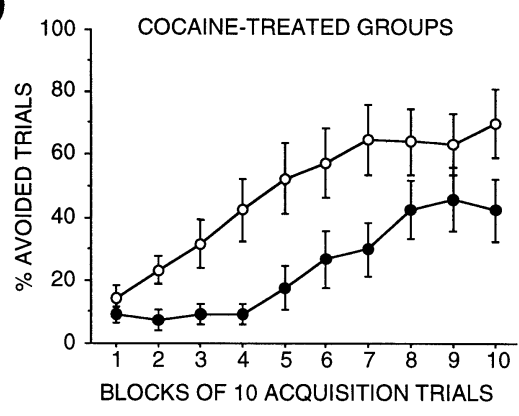


$[F(1,14)=24.7, P<0.001]$ and a significant interaction of Pre-exposure condition $\times$ Blocks $[F(9,126)=3.2, P<0.002]$. A similar analysis for the VEH groups revealed neither a significant effect of Pre-exposure condition $(P=0.32)$ nor a Pre-exposure condition $\times$ Blocks interaction $(P=0.53)$. Numbers of non-escaped trials and inter-trial interval shuttles made during the avoidance test session did not differ as a function of either Drug treatment or Pre-exposure condition (data not shown).

\section{Cocaine withdrawal}

A comparison of the number of crossings made during the two shuttle box pre-exposure sessions (Table 1) again revealed a significant effect of Session $[F(1,44)=$ $34.1, P<0.0001]$, reflecting a generally higher level of activity on the first pre-exposure day compared to the second day. There was also a significant main effect of CS Pre-exposure condition $[F(1,44)=9.9, P<0.01]$ due to the decreased number of shuttles made by PE compared to NPE animals. Shuttles made during the pre-exposure sessions did not differ between cocaine-treated (COC) and VEH animals, regardless of Pre-exposure condition.

An overall $2 \times 2 \times 10$ ANOVA (Drug treatment $\times$ Pre-exposure condition $\times$ Blocks of ten trials) of percentage avoidance responses during test (Fig. 1B, D) yielded a highly significant main effect of Blocks $[F(9,396)=37.6$, $P<0.001]$ reflecting increasing numbers of successfully avoided trials throughout the test session. A significant main effect of Pre-exposure condition $[F(1,44)=6.7$, $P<0.015]$ was noted as well. Although there was no significant Drug $\times$ Pre-exposure interaction in this analysis, the main effect of CS pre-exposure was almost entirely due to PE versus NPE differences in the COC-treated groups. This observation was confirmed by separate $2 \times 10$ ANOVAs (Pre-exposure $\times$ Blocks of ten trials) within each of the drug treatment conditions. Although a significant main effect of Pre-exposure condition $[F(1,22)=6.3, \quad P<0.05]$ was determined for the COC groups, indicating significant latent inhibition, no effects of CS pre-exposure were found for the VEH groups (no main effect of Pre-exposure condition: $P=0.31$; no Preexposure condition $\times$ Blocks interaction: $P=0.93$ ). Numbers of non-escaped trials and inter-trial interval shuttles made during the avoidance test session did not differ as a function of either Drug treatment or Pre-exposure condition (data not shown).

Experiment 2: effects of psychostimulant withdrawal on prepulse inhibition of acoustic startle

\section{Amphetamine withdrawal}

Startle response amplitudes (Fig. 2A) were analyzed with two-way repeated-measures ANOVAs (Drug treatment $\times$ Trials). Startle response amplitudes habituated
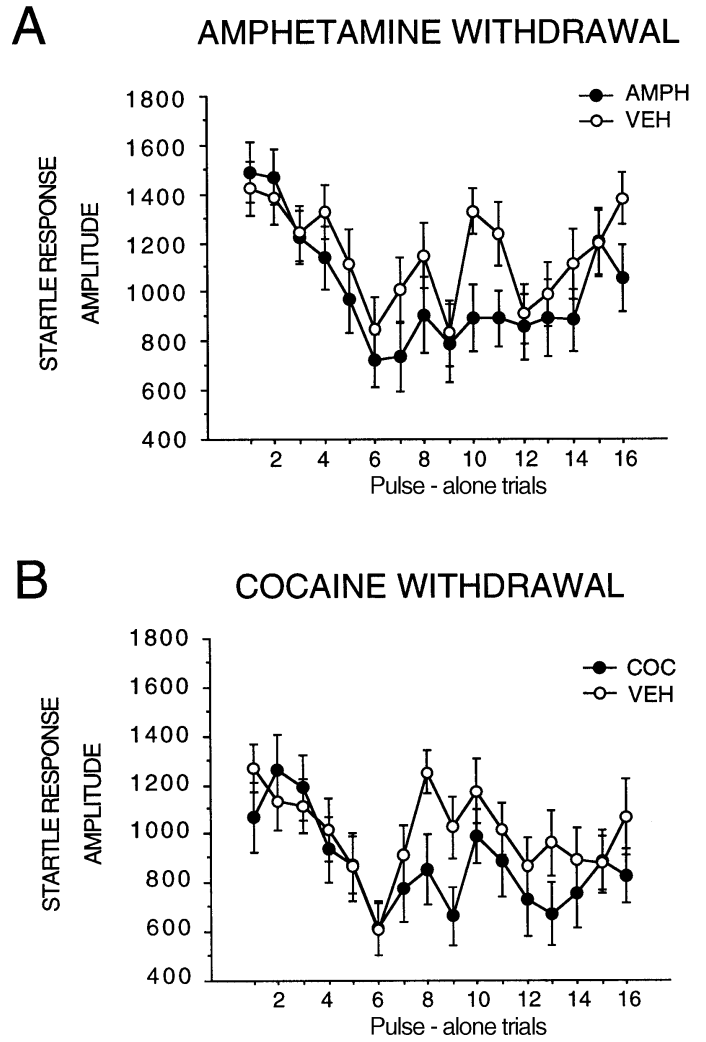

Fig. 2 Startle responses during 16 pulse-alone trials in the prepulse inhibition test in animals previously treated with five daily injections of $\mathbf{A}$ amphetamine $(A M P H), \mathbf{B}$ cocaine $(C O C)$ or $\mathbf{A}, \mathbf{B}$ saline vehicle $(V E H)$. All animals were tested 4 days after their last drug or vehicle injection. $n=8$ per group. Values are means \pm SEM

similarly for the two drug treatment conditions during the first four "warm-up" presentations of the startle pulse [ $2 \times 4$ ANOVA, main effect of Trial: $F(3,90)=3.4$, $P<0.05]$. Although visual inspection of the data suggests that the AMPH group exhibited somewhat dampened startle responses during the latter part of the test session, a $2 \times 12$ ANOVA of the last 12 startle pulse presentations did not detect a significant effect of Drug pretreatment [non-significant effect of Drug: $F(1,30)=1.77, P=0.19$ ] A $2 \times 4$ ANOVA (Drug treatment $\times$ Prepulse intensity) revealed a significant main effect of Prepulse intensity on inhibition of the startle response $[F(1,90)=44.9$, $P<0.0001]$ such that prepulses of greater intensity produced stronger response inhibition (Fig. 3A). However, percentage PPI was not influenced by prior history of AMPH treatment, as no significant differences were observed between AMPH and VEH groups.

\section{Cocaine withdrawal}

An analysis of startle response amplitudes (Fig. 2B) did not reveal significant differences between the responses of the COC and VEH groups to the acoustic startle stimulus. Startle response amplitudes during the first four 
A AMPHETAMINE WITHDRAWAL
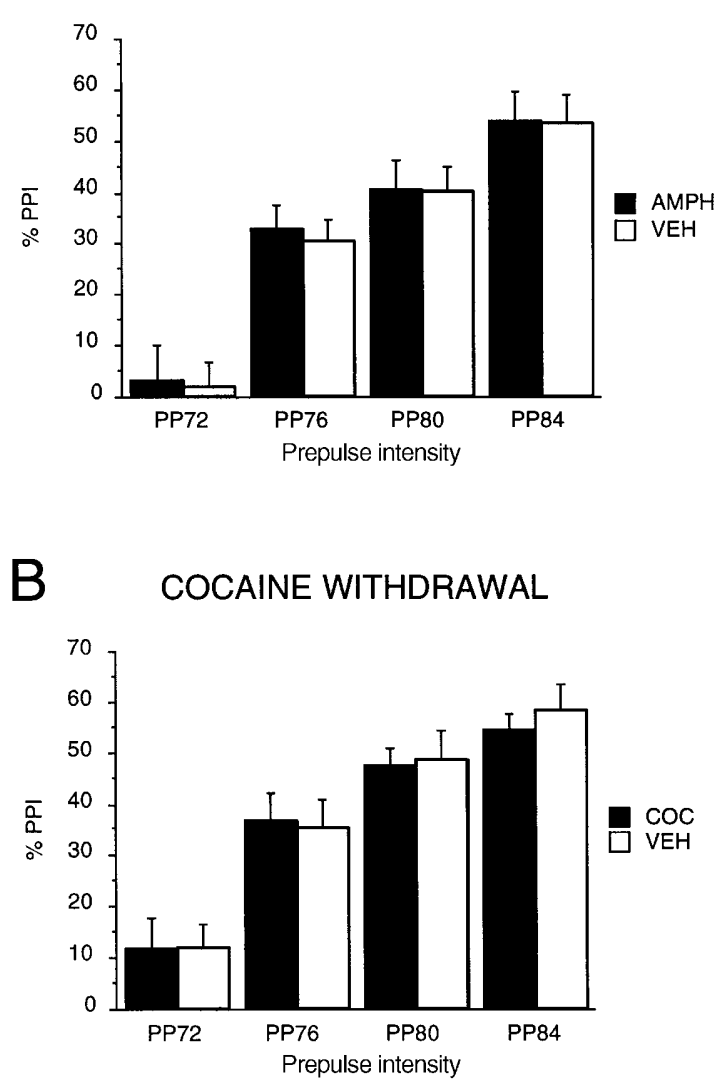

Fig. 3 Percentage prepulse inhibition $(P P I)$ of the acoustic startle response in animals previously treated with $\mathbf{A}$ amphetamine $(A M P H), \mathbf{B}$ cocaine $(C O C)$ or $\mathbf{A}, \mathbf{B}$ saline vehicle $(V E H)$, measured using a range of prepulse intensities $(72,76,80$ or $84 \mathrm{~dB}$ [A]) 4 days after their last injection. $n=8$ per group. Values are means \pm SEM

"warm-up" pulse presentations showed the expected response habituation $[2 \times 4$ ANOVA, main effect of Trials: $F(3,90)=2.9, P<0.05]$ and did not differ between drug treatment groups. However, similarly to the AMPH withdrawal experiment, COC animals demonstrated a nonsignificant reduction in startle response amplitudes to the pulse-alone trials to the last 12 startle pulse presentations [non-significant effect of Drug: $F(1,30)=2.11, P=0.16$ ] A two-way ANOVA (Drug treatment $\times$ Prepulse intensity) performed on percentage PPI did not detect any differences between the COC and VEH groups (Fig. 3B); as expected, however, there was again a significant effect of the factor of prepulse intensities $[F(3,90)=54.8$, $P<0.0001]$ reflecting a gradual increase in PPI as a function of the intensity of the prepulse stimulus.

\section{Locomotor sensitization to psychostimulants}

Animals used in experiment 1 were tested on day 5 of the drug withdrawal period for their locomotor response to a challenge injection of either AMPH or COC (AMPH
A

AMPHETAMINE WITHDRAWAL

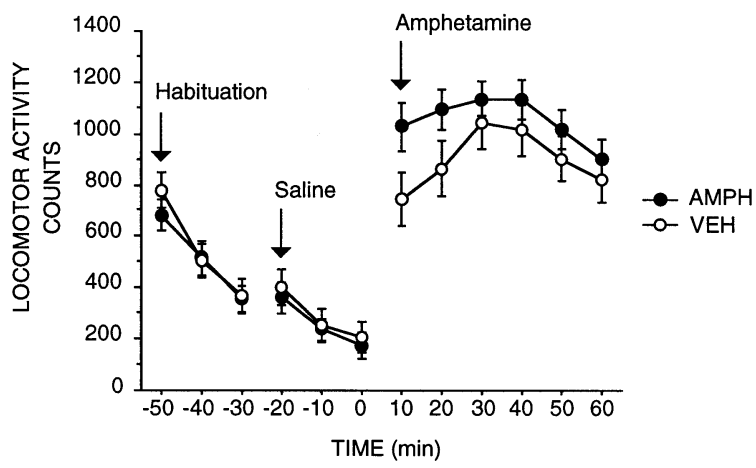

B

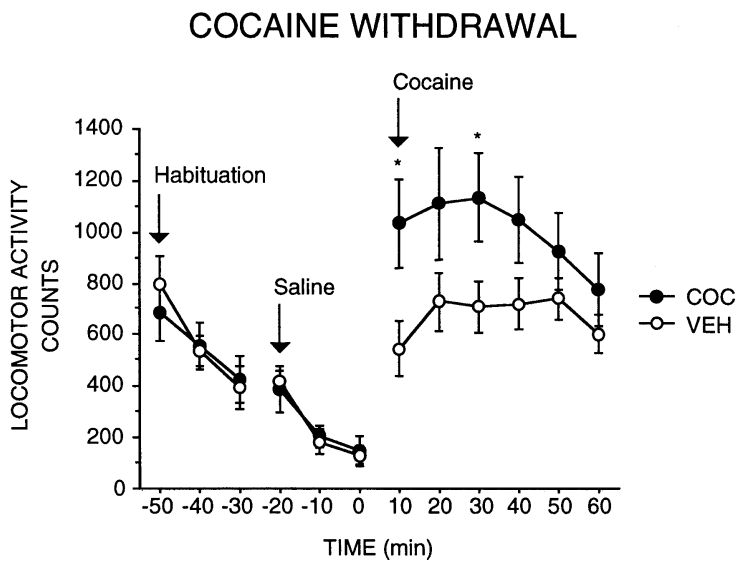

Fig. 4 Locomotor activity measured during an initial 30-min period in a novel open field environment, a 30-min period following an injection of saline vehicle, and a 1-h period following a challenge injection with either A amphetamine $(1.5 \mathrm{mg} / \mathrm{kg})$ or $\mathbf{B}$ cocaine hydrochloride $(20 \mathrm{mg} / \mathrm{kg}$, IP) in animals previously treated over 5 days with amphetamine $(A M P H)$, cocaine $(C O C)$ or saline vehicle $(V E H)$. All animals were tested 5 days after their last drug or vehicle injection. Amphetamine experiment: $n=8$ per group. Cocaine experiment: $n=4$ per group. ${ }^{*} P<0.05$ compared to $\mathrm{VEH}$ group. Values are means \pm SEM

withdrawal experiment: $n=16$ per drug condition, using pooled PE and NPE groups; COC withdrawal experiment: only half of the animals were tested, i.e. $n=8$ per drug condition, using pooled PE and NPE groups; see Fig. 4). Analyses using $2 \times 3$ ANOVAs (Drug treatment $\times 10$-min Blocks) revealed no significant effects of either prior COC or AMPH treatment on locomotor activity measured during either the 30-min habituation period or during the 30-min period which followed an injection of saline vehicle, although highly significant effects of Blocks were detected for both sessions which reflect the normal reductions in locomotion that follow placement into a novel environment [AMPH withdrawal experiment: habituation period $-F(2,60)=60.35, P<0.0001$; saline injection period $-F(2,60)=17.89, P<0.0001$; COC withdrawal experiment: habituation period $-F(2,28)=$ $17.65, P<0.0001$; saline injection period $-F(2,28)=$ 41.27, $P<0.0001]$. Both the COC- and AMPH-treated 
groups demonstrated a modest degree of behavioral sensitization to a challenge injection of drug. A $2 \times 6 \mathrm{AN}-$ OVA (Drug treatment $\times 10$-min Blocks) revealed significant main effects of Blocks for both AMPH- and COCpretreated rats [AMPH withdrawal experiment: $F(5,150)=10.23, P<0.0001$; COC withdrawal experiment: $F(5,70)=4.83, P<0.001]$. AMPH-withdrawn animals demonstrated increased locomotor activity compared to the VEH group, particularly during the first 20 min following administration of a challenge injection of AMPH, an effect which approached statistical significance [Drug $\times$ Blocks interaction: $F(5,150)=2.04$, $P=0.076]$. Similarly, a significant Drug $\times$ Blocks interaction $[F(5,70)=2.54, \quad P<0.05]$ for the COC-challenged groups reflected the increased locomotor response of COC-withdrawn animals to subsequent COC administration compared to the response of animals previously injected with VEH. Half of the animals from experiment 2 were also tested for locomotor sensitization to a challenge drug injection one day after PPI testing (data not shown). Activity testing of these animals was carried out in an open field in combination with the Noldus Ethovision system (Wageningen, Netherlands) rather than the infrared-detection locomotor activity monitors used for animals in Experiment 1; consequently, sensitization data from experiments 1 and 2 are not directly comparable. Nevertheless, PPI-tested rats withdrawn from AMPH showed non-significantly increased locomotor responding to a drug challenge [main effect of Drug: $F(1,14)=2.66, P=0.12 ; n=8$ per group, data not shown], and PPI-tested rats withdrawn from COC showed increased activity which was greatest during the first $10 \mathrm{~min}$ after drug injection [Drug $\times$ Blocks interaction: $F(5,70)=3.6, P<0.01 ; n=8$ per group, data not shown].

\section{Discussion}

The results of the present study indicate that animals recently withdrawn from repeated daily COC or AMPH administration exhibit enhanced latent inhibition (LI) of two-way active avoidance acquisition. These results are consistent with those of an earlier investigation on the effects of chronic AMPH on LI in a conditioned emotional response paradigm (Weiner et al. 1984), in which LI was non-significantly increased in animals after 1-2 days of withdrawal from 14 days of amphetamine treatment. A similar enhancement of LI of conditioned freezing behavior has also been observed following the chronic cocaine regimen used in this study (Murphy et al. 2001). In contrast to these effects on LI, PPI was unaffected by either prior AMPH or COC treatment.

The increased LI observed following AMPH and COC withdrawal reflects both reduced avoidance in the PE groups and improved avoidance in the NPE groups. Moreover, this effect was recorded on a background of no LI in the VEH-treated animals. The absence of LI in the VEH control groups was somewhat surprising, given the high number of CS pre-exposures that preceded con- ditioned avoidance testing. Recent experiments in our laboratory have in fact replicated these results using fewer CS pre-exposures, again finding no LI in controls and enhanced LI in AMPH-withdrawn animals (Russig, Murphy and Feldon, unpublished findings). The expression of LI is said to be critically dependent on a number of different conditioning parameters, as well as general factors such as environmental stress (Hellman et al. 1983; Hall 1991; Killcross and Balleine 1996). Thus, although it is not clear why the parameters for eliciting LI vs. no LI may vary somewhat between experiments, the effects of psychostimulant withdrawal in enhancing LI under conditions in which LI is absent in the VEH groups appear to be highly replicable.

Short-term psychostimulant withdrawal is claimed to induce dysphoric behaviors in rats (Leith and Barrett 1976; Markou and Koob 1991; Barr and Phillips 1999; Barr et al. 1999). Given that the learned helplessness theory of depression would predict deficits in active avoidance and/or shock escape (McKinney 1984), it is somewhat surprising that NPE-COC and NPE-AMPH subjects in fact tended to avoid footshocks more successfully than NPE-saline animals and experienced similar numbers of non-escaped trials. However, both AMPH and $\mathrm{COC}$ were administered at relatively low doses and over a brief period in this study. Demonstrations of depressed reward sensitivity during psychostimulant withdrawal are typically observed after the administration of higher doses over a more extended period of time and are also somewhat transient, such that these effects, if they did exist, might be diminished even after only 4 days of withdrawal. In contrast, rats which have been withdrawn from low-dose drug regimens similar to those used in this study have been shown to exhibit enhanced conditioned approach behavior and conditioned inhibition (Harmer and Phillips 1999) as well as behavioral signs of anxiety and arousal in the face of environmental stressors (Harris and Aston-Jones 1993; Sarnyai et al. 1995; Borowski and Kokkinidis 1998; Fiorino and Phillips 1999). The presence of increased anxiety in stimulant-sensitized NPE subjects during withdrawal might contribute to the somewhat improved avoidance behavior demonstrated by these groups. That is, an increased state of arousal in these groups could either heighten their negative experience of the shock or lead to enhanced attention to their environment, both effects which might be expected to increase conditioned avoidance learning. However, assuming that such a state exists for the NPE rats, it follows that the AMPH-PE and COC-PE rats would be similarly influenced; instead, their avoidance performance was non-significantly decreased with respect to that of VEH controls.

The reduced avoidance acquisition of the drug-PE groups may reflect a kind of cognitive inflexibility, or an inability to switch from one learned contingency (tone $\mathrm{CS}$ - nothing) to another (tone CS - shock). Interestingly, Kokkinidis (1983) reported that while mice recently withdrawn from AMPH do not show an active avoidance acquisition deficit, they do exhibit reduced extinction of 
the avoidance response to a CS no longer paired with footshock, perhaps representing a similar type of cognitive perseveration. Observations of enhanced LI in patients with obsessive-compulsive disorder (OCD; Swerdlow et al. 1999) are also consistent with this interpretation. Thus, the co-occurrence of increased anxiety and increased perseverative behavior in the stimulant-withdrawn groups might explain the bi-directional effects of withdrawal on avoidance in NPE and PE rats.

Recent in vivo microdialysis investigations have confirmed the importance of nucleus accumbens DA in the expression of LI (Young et al. 1993; Murphy et al. 2001). Some investigators have suggested that excessive DA activity in the nucleus accumbens results in rapid switching from one stimulus-response pattern to another, and that such a mechanism is responsible for the disruption of LI in animals treated with acute amphetamine (Van den Bos and Cools 1989; Weiner and Feldon 1997). Therefore, enhanced LI might conversely reflect reduced switching and reduced nucleus accumbens DA. The increased LI found in the present study during COC and AMPH withdrawal resembles the previously reported potentiation of LI which occurs when DA-antagonist antipsychotic drugs are administered prior to pre-exposure and conditioning sessions (Weiner and Feldon 1987; Dunn et al. 1993; Weiner et al. 1996). There is evidence that the effects of antipsychotic drugs on LI are mediated in the nucleus accumbens (Gray et al. 1997); furthermore, reports of reduced DA function in the nucleus accumbens during COC and AMPH withdrawal (Parsons et al. 1991; Rossetti et al. 1992; Weiss et al. 1992, 1997; Kuhar and Pilotte 1996) are consistent with the idea that enhanced LI reflects DA antagonism in mesolimbic targets during pre-exposure and/or avoidance testing. Nevertheless, reductions in nucleus accumbens DA during psychostimulant withdrawal are not reported by all laboratories and may be dependent on schedules of drug administration or procedural details (Crippens et al. 1993; Crippens and Robinson 1994); in particular, it is very possible that the schedule of low-dose AMPH employed in this study would not have been sufficient to reduce accumbens DA during withdrawal. Finally, in an earlier study in which COC was administered subsequent to CS pre-exposure and CS-UCS conditioning, we also observed enhanced LI of conditioned freezing behavior during COC withdrawal (Murphy et al. 2001). This earlier result suggests that neuroleptic-like effects need not be experienced during pre-exposure and conditioning to elicit enhanced LI at test. Therefore, the effects of psychostimulant withdrawal on LI may affect processes of retrieval rather than acquisition (Kraemer and Spear 1992; Killcross et al. 1998), such that the neurolepticlike potentiation of LI in this study may be merely coincidental.

In contrast to the effects of short-term withdrawal from psychostimulants on LI, drug and VEH groups exhibited similar levels of PPI of the acoustic startle response as well as similar degrees of startle during the pulse-alone trials. The PPI result is consistent with previous reports which found no lasting effects of prior repeated AMPH, COC or apomorphine exposure on PPI when animals were subsequently tested in either a drug-free state or following a challenge injection of drug (Mansbach et al. 1988; Druhan et al. 1998; Martin-Iverson 1999; Martinez et al. 1999); in fact, one study found that chronic COC treatment eliminated rather than augmented the normal deficit produced by acute COC pretreatment (Byrnes and Hammer 2000). Although LI and PPI are believed to reflect similar aspects of cognitive processing, disparities in the regulation of these phenomena have previously been reported (Wilkinson et al. 1994; Feldon et al. 2000). The brain nuclei believed to mediate LI and PPI are also not identical; for example, whereas the prefrontal cortex has consistently been implicated in the mediation of PPI, prefrontal cortical manipulations do not influence LI (Ellenbroek et al. 1996; Lacroix et al. 1998; Broersen et al. 1999). An additional consideration stems from the potential contribution of the discrepant VEH-treated groups' baselines (no LI versus presence of PPI) to the relative ability of stimulant withdrawal to elicit enhancements in these two paradigms. However, given the low to moderate PPI exhibited by the VEH groups in this study, we feel that either an enhancement or disruption in PPI would have been observable under the conditions used here; therefore, the differential VEH-group baselines would not have contributed significantly to the differential effects of stimulant withdrawal on LI versus PPI. Nevertheless, there is evidence that PPI is generally somewhat more resistant to enhancement (Depoortere et al. 1997a, 1997b) than LI, a concern which may contribute to the lack of effect of psychostimulant withdrawal on PPI observed in the present study.

Although we did see evidence of locomotor sensitization to a challenge injection of COC or AMPH administered on day 5 of withdrawal, the activity levels of stimulant-sensitized animals were not markedly greater than those of saline-treated controls. Behavioral sensitization to psychostimulants is at least partially context-dependent (Post et al. 1987; Badiani et al. 1995); therefore, the fact that locomotor activity in response to a drug challenge was only slightly greater in the AMPH and COC groups compared to the VEH groups in this study may be because animals were placed in their home cages after each injection, but tested for sensitized locomotor responding in a novel environment.

In conclusion, short-term withdrawal from repeated administration of low-dose AMPH and COC did not disrupt either LI or PPI; instead, LI was enhanced and PPI was unaffected by the treatment regimens used in this study. Thus, withdrawal from psychostimulants does not produce cognitive deficits consistent with modelling of the positive symptoms of schizophrenia, despite the relationship that is believed to exist between this class of symptoms and sensitized behavioral responding to a drug challenge. It remains possible that a more intensive psychostimulant pretreatment than the one used in this study would induce a different pattern of cognitive ef- 
fects than the ones we observed; thus, further research is warranted to examine the effects of higher dose schedules of AMPH and COC administration on these behaviors. The available literature suggests that the effects of psychostimulant withdrawal are consistent with symptoms of anhedonia and anxiety, or even negative schizophrenic symptoms (i.e. blunted affect, apathy and cognitive disorganization; Andreasen et al., 1995), rather than the positive symptoms of the disease. Therefore, the enhanced LI that we report here may be a sign of cognitive phenomena more closely related to these psychological conditions. The findings of the present study suggest that examination of the consequences of short-term stimulant withdrawal in the rat may contribute to our understanding of neural mechanisms underlying specific deficits in cognitive processing and altered emotional states.

Acknowledgements We would like to thank the staff of the animal facility for their care and maintenance of the animals used in this study and Mr. Peter Schmid for his excellent technical assistance. Support for this project was provided by the Swiss Federal Institute of Technology (ETH-Zurich).

\section{References}

Andreasen NC, Arndt S, Alliger R, Miller D, Flaum M (1995) Symptoms of schizophrenia: methods, meanings and mechanisms. Arch Gen Psychiatry 15:341-351

Badiani A, Browman KE, Robinson TE (1995) Influence of novel versus home environment on sensitization to the psychomotor stimulant effects of cocaine and amphetamine. Brain Res 674:291-298

Barr AM, Phillips AG (1999) Withdrawal following repeated exposure to $d$-amphetamine decreases responding for a sucrose solution as measured by a progressive ratio schedule of reinforcement. Psychopharmacology 141:99-106

Barr AM, Fiorino DF, Phillips AG (1999) Effects of withdrawal from an escalating dose schedule of $d$-amphetamine on sexual behavior in the male rat. Pharmacol Biochem Behav 64: 597-604

Baruch I, Hemsley DR, Gray JA (1988) Differential performance of acute and chronic schizophrenics in a latent inhibition task. J Nerv Ment Dis 176:598-606

Borowski TB, Kokkinidis L (1998) The effects of cocaine, amphetamine, and the dopamine $\mathrm{D}_{1}$ receptor agonist SKF 38393 on fear extinction as measured with potentiated startle: implications for psychomotor stimulant psychosis. Behav Neurosci 112:952-965

Brady KT, Lydiard RB, Malcolm R, Ballenger JC (1991) Cocaineinduced psychosis. J Clin Psychiatry 52:509-512

Braff DL, Geyer MA (1990) Sensorimotor gating and the neurobiology of schizophrenia: human and animal model studies. Arch Gen Psychiatry 47:181-188

Braff DL, Stone C, Callaway E, Geyer M, Glick I, Bali L (1978) Prestimulus effects on human startle reflex in normals and schizophrenics. Psychophysiology 15:339-343

Broersen LM, Feldon J, Weiner I (1999) Dissociative effects of apomorphine infusions into the medial prefrontal cortex of rats on latent inhibition, prepulse inhibition and amphetamine-induced locomotion. Neuroscience 94:39-46

Byrnes JJ, Hammer RP Jr (2000) The disruptive effect of cocaine on prepulse inhibition is prevented by repeated administration in rats. Neuropsychopharmacology 22:551-554

Crippens D, Robinson TE (1994) Withdrawal from morphine or amphetamine: different effects on dopamine in the ventral-medial striatum studies with microdialysis. Brain Res 650:56-62
Crippens D, Camp DM, Robinson TE (1993) Basal extracellular dopamine in the nucleus accumbens during amphetamine withdrawal: a "no net flux" microdialysis study. Neurosci Lett 164:145-148

Depoortere R, Perrault G, Sanger DJ (1997a) Potentiation of prepulse inhibition of the startle reflex in rats: pharmacological evaluation of the procedure as a model for detecting antipsychotic activity. Psychopharmacology 132:366-374

Depoortere R, Perrault G, Sanger, DJ (1997b) Some, but not all, antipsychotic drugs potentiate a low level of prepulse inhibition shown by rats of the Wistar strain. Behav Pharmacol 8:364-372

Druhan JP, Geyer MA, Valentino RJ (1998) Lack of sensitization to the effects of $d$-amphetamine and apomorphine on sensorimotor gating in rats. Psychopharmacology 135:296-304

Dunn LA, Atwater GE, Kilts CD (1993) Effects of antipsychotic drugs on latent inhibition/sensitivity and specificity of an animal behavioral model of clinical drug action. Psychopharmacology 112:315-323

Ellenbroek BA, Budde S, Cools AR (1996) Prepulse inhibition and latent inhibition: the role of dopamine in the medial prefrontal cortex. Neuroscience 75:535-542

Feldon J, Lehmann J, Pryce C, Weiss I (2000) Rat latent inhibition and prepulse inhibition are sensitive to different manipulations of the social environment: a comprehensive study of the environmental approach to neurodevelopmental models of schizophrenia. In: Myslobodsky M, Weiner I (eds) Contemporary issues in modelling psychopathology. Kluwer, Boston, pp 231-245

Fiorino DF, Phillips AG (1999) Facilitation of sexual behavior in male rats following $d$-amphetamine-induced behavioral sensitization. Psychopharmacology 142:200-208

Gawin FH (1991) Cocaine addiction: Psychology and neurophysiology. Science 251:1580-1586

Gawin FH, Kleber HD (1986) Abstinence symptomatology and psychiatric diagnosis in cocaine abusers: clinical observations. Arch Gen Psychiatry 43:107-113

Geyer MA, Swerdlow NL, Mansbach RS, Braff DL (1990) Startle response models of sensorimotor gating and habituation deficits in schizophrenia. Brain Res Bull 25:485-498

Graham FK (1975) The more or less startling effects of weak prestimulation. Psychophysiology 12:238-248

Gray JA, Moran PM, Grigoryan G, Peters SL, Young AMJ, Joseph MH (1997) Latent inhibition: the nucleus accumbens connection revisited. Behav Brain Res 88:27-34

Gray NS, Hemsley DR, Gray JA (1992) Abolition of latent inhibition in acute, but not chronic schizophrenics. Neurol Psychiatry Brain Res 88:27-34

Gray NS, Pilowsky LS, Gray JA, Kerwin RW (1995) Latent inhibition in drug naive schizophrenics: relationship to duration of illness and dopamine $\mathrm{D}_{2}$ binding using SPET. Schizophr Res 17:95-107

Hall G (1991) Perceptual and associative learning. Oxford University Press, Oxford

Harmer CJ, Phillips GD (1999) Enhanced conditioned inhibition following repeated pretreatment with $d$-amphetamine. Psychopharmacology 142:120-131

Harris GC, Aston-Jones G (1993) $\beta$-Adrenergic antagonists attenuate withdrawal anxiety in cocaine and morphine-dependent rats. Psychopharmacology 113:131-136

Hellman PA, Crider A, Solomon PR (1983) Interaction of tailpressure stress and $d$-amphetamine in disruption of the rat's ability to ignore an irrelevant stimulus. Behav Neurosci 97: 1017-1021

Hoffman HS, Ison JR (1980) Reflex modification in the domain of startle: I. Some empirical findings and their implications for how the nervous system processes sensory input. Psychol Rey $87: 175-189$

Imperato A, Mele A, Scrocco MG, Puglisi-Allegra S (1992) Chronic cocaine alters limbic extracellular dopamine. Neurochemical basis for addiction. Eur J Pharmacol 212:299-300

Killcross AS, Balleine B (1996) Role of primary motivation in stimulus pre-exposure effects. J Exp Psychol 22:32-42 
Killcross AS, Kiernan MJ, Dwyer D, Westbrook RF (1998) Effects of retention interval on latent inhibition and perceptual learning. Q J Exp Psychol 51B:59-74

Koch M, Schnitzler H-U (1997) The acoustic startle response in rats - circuits mediating evocation, inhibition and potentiation. Behav Brain Res 89:35-49

Kokkinidis L (1983) The effects of chronic amphetamine administration on the acquisition and extinction of an active and passive avoidance response in mice. Pharmacol Biochem Behav 19:593-598

Koob GF, Bloom FE (1988) Cellular and molecular mechanisms of drug dependence. Science 242:715-723

Kraemer PJ, Spear NE (1992) The effect of nonreinforced stimulus exposure on the strength of a conditioned taste aversion as a function of retention interval: do latent inhibition and extinction involve a shared process? Anim Learn Behav 20:1-7

Kuhar MJ, Pilotte NS (1996) Neurochemical changes in cocaine withdrawal. Trends Pharmacol Sci 17:260-263

Lacroix L, Broersen LM, Weiner I, Feldon J (1998) The effects of excitotoxic lesion of the medial prefrontal cortex on latent inhibition, prepulse inhibition, food hoarding, elevated plus maze, active avoidance and locomotor activity in the rat. Neuroscience 82:431-442

Lago JA, Kosten TR (1994) Stimulant withdrawal. Addiction 89:1477-1481

Leith NJ, Barrett RJ (1976) Amphetamine and the reward system: evidence for the tolerance and the post-drug depression. Psychopharmacology 46:19-25

Lubow RE (1973) Latent inhibition. Psychol Bull 79:398-407

Mansbach RS, Geyer MA, Braff DL (1988) Dopaminergic stimulation disrupts sensorimotor gating in the rat. Psychopharmacology 94:507-514

Markou A, Koob GF (1991) Postcocaine anhedonia: an animal model of cocaine withdrawal. Neuropsychopharmacology 4: $17-25$

Martin-Iverson MT (1999) Does sensitization occur to prepulse inhibition of the startle reflex effects of repeated apomorphine treatments in rats? J Psychopharmacology 13:261-273

Martinez ZA, Ellison GD, Geyer MA, Swerdlof NR (1999) Effects of sustained cocaine exposure on sensorimotor gating of startle in rats. Psychopharmacology 142:253-260

McKinney WT (1984) Animal models of depression: an overview. Psychiatr Dev 2:77-96

Murphy CA, Pezze M-A, Feldon J, Heidbreder C (2000) Differential involvement of dopamine in the shell and core of the nucleus accumbens in the expression of latent inhibition to an aversively-conditioned stimulus. Neuroscience 97:469-477

Murphy CA, Heidbreder C, Feldon J (2001) Acute withdrawal from repeated cocaine treatment enhances latent inhibition of a conditioned fear response. Behav Pharmacology 12:13-23

Parsons LH, Smith AD, Justice JB Jr (1991) Basal extracellular dopamine is decreased in the rat nucleus accumbens during abstinence from chronic cocaine. Synapse 9:60-65

Pierce RC, Kalivas PW (1997) A circuitry model of the expression of behavioral sensitization to amphetamine-like psychostimulants. Brain Res Rev 25:192-216

Post RM (1975) Cocaine psychosis: a continuum model. J Psychiatry 132:225-231

Post RM, Weiss SRB, Pert A (1987) The role of context and conditioning in behavioral sensitization to cocaine. Psychopharmacology Bull 23:425-429
Robinson TE, Becker JB (1986) Enduring changes in brain and behavior produced by chronic amphetamine administration: a review and evaluation of animal models of amphetamine psychosis. Brain Res Rev 11:157-198

Rossetti ZL, Hmaidan Y, Gessa GL (1992) Marked inhibition of mesolimbic dopamine release: a common feature of ethanol, morphine, cocaine and amphetamine abstinence in rats. Eur J Pharmacol 221:227-234

Sarnyai Z, Biro E, Gardi J, Vecsernyes M, Julesz J, Telegdy G (1995) Brain corticotropin-releasing factor mediates "anxietylike" behavior induced by cocaine withdrawal in rats. Brain Res 675:89-97

Snyder SH (1973) Amphetamine psychosis: a "model" schizophrenia mediated by catecholamines. Am J Psychiatry 130: 61-67

Swerdlow NR, Braff DL, Geyer MA, Caine SB (1992) The neural substrates of sensorimotor gating of the startle reflex: a review of recent findings and their implications. J Psychopharmacol $6: 176-190$

Swerdlow NR, Braff DL, Hartson H, Perry W, Geyer MA (1996) Latent inhibition in schizophrenia. Schizophr Res 20:91-103

Swerdlow NR, Hartston HJ, Hartman PL (1999) Enhanced visua latent inhibition in obsessive-compulsive disorder. Biol Psychiatry 45:482-488

Taylor MK, Ison JR, Schwarzkopf SB (1995) Effects of single and repeated exposure to apomorphine on the acoustic startle reflex and its inhibition by a visual prepulse. Psychopharmacology 120:117-127

Van den Bos R, Cools AR (1989) The involvement of the nucleus accumbens in the ability of rats to switch to cue-induced behaviors. Life Sci 44:1697-1704

Weiner I, Feldon J (1987) Facilitation of latent inhibition by haloperidol in rats. Psychopharmacology 91:248-53

Weiner I, Feldon J (1997) The switching model of latent inhibition: an update of neural substrates. Behav Brain Res 88:11-25

Weiner I, Lubow RE, Feldon J (1984) Abolition of the expression but not the acquisition of latent inhibition by chronc amphetamine in rats. Psychopharmacology 83:194-199

Weiner I, Shadach E, Tarrasch R, Kidron R, Feldon J (1996) The latent inhibition model of schizophrenia: further validation using the atypical neuroleptic, clozapine. Biol Psychiatry 593: 314-318

Weiss F, Markou A, Lorang MT, Koob GF (1992) Basal extracellular dopamine levels in the nucleus accumbens are decreased during cocaine withdrawal after unlimited-access self-administration. Brain Res 593:314-328

Weiss F, Imperato A, Casu MA, Mascia MS, Gessa GL (1997) Opposite effects of stress on dopamine release in the limbic system of drug-naive and chronically amphetamine-treated rats. Eur J Pharmacol 337:219-222

Wilkinson LS, Killcross SS, Humby T, Hall FS, Geyer MA, Robbins TW (1994) Social isolation in the rat produces developmentally specific deficits in prepulse inhibition without disrupting latent inhibition. Neuropsychopharmacology 10:61-72

Young AMJ, Joseph MH, Gray JA (1993) Latent inhibition of conditioned dopamine release in rat nucleus accumbens. Neuroscience 54:5-9

Zhang J, Engel JA, Soderpalm B, Svensson L (1998) Repeated administration of amphetamine induces sensitization to its disruptive effect on prepulse inhibition in the rat. Psychopharmacology 135:401-406 